\title{
Long-Range Correlated Dynamics in Intrinsically Disordered Proteins
}

Giacomo Parigi, ${ }^{\dagger, \nabla}$ Nasrollah Rezaei-Ghaleh, ${ }^{\ddagger}, \S, \nabla$ Andrea Giachetti, ${ }^{\dagger}$ Stefan Becker, ${ }^{\S}$ Claudio Fernandez, ${ }^{\perp}$ Martin Blackledge," Christian Griesinger, ${ }^{\S}$ Markus Zweckstetter, ${ }^{*}, \S, \#$ and Claudio Luchinat* ${ }^{* \dagger}$

${ }^{\dagger}$ Department of Chemistry “Ugo Schiff” and CERM, University of Florence, via Sacconi 6, 50019 Sesto Fiorentino, Italy

${ }^{\ddagger}$ German Center for Neurodegenerative Diseases (DZNE), Am Fassberg 11, 37077 Göttingen, Germany

${ }^{\S}$ Department for NMR-based Structural Biology, Max Planck Institute for Biophysical Chemistry, Am Fassberg 11, 37077 Göttingen, Germany

${ }^{\perp}$ Max Planck Laboratory of Structural Biology, Chemistry and Molecular Biophysics of Rosario (MPLbioR), Drug Discovery Unit (SEDIPFAR), National University of Rosario and CONICET Rosario, S2000CKG Rosario, Santa Fe, Argentina

"CNRS, Protein Dynamics and Flexibility, Institut de Biologie Structurale, 38000 Grenoble, France

${ }^{\#}$ Center for Nanoscale Microscopy and Molecular Physiology of the Brain (CNMPB), University Medical Center, 37073 Göttingen, Germany

\section{Supporting Information}

ABSTRACT: Intrinsically disordered proteins (IDPs) are involved in a wide variety of physiological and pathological processes and are best described by ensembles of rapidly interconverting conformers. Using fast field cycling relaxation measurements we here show that the IDP $\alpha$-synuclein as well as a variety of other IDPs undergoes slow reorientations at time scales comparable to folded proteins. The slow motions are not perturbed by mutations in $\alpha$-synuclein, which are related to genetic forms of Parkinson's disease, and do not depend on secondary and tertiary structural propensities. Ensemble-based hydrodynamic calculations suggest that the time scale of the underlying correlated motion is largely determined by hydrodynamic coupling between locally rigid segments. Our study indicates that long-range correlated dynamics are an intrinsic property of IDPs and offers a general physical mechanism of correlated motions in highly flexible biomolecular systems.

\section{INTRODUCTION}

Intrinsically disordered proteins (IDPs) are prevalent in eukaryotic proteomes and mediate key roles in biological processes including protein biosynthesis, cell cycle regulation, and signal transduction. ${ }^{1}$ Due to their connection to disease states such as cancer, neurodegeneration, and cardiovascular diseases, there is growing interest in the structural and dynamic characterization of IDPs as potential pharmacological targets. ${ }^{2}$

IDPs are best described as heterogeneous structural ensembles, as there is no single strongly favored structure in their conformational energy landscapes. ${ }^{3}$ Through combination of experimental and computational methods, description of structural properties of IDPs advanced in recent years from the detection of secondary and tertiary structural propensities to atomic level representations of the conformational ensembles. $^{4,5}$ In parallel, backbone motions of disordered proteins were studied through high-resolution NMR techniques, especially ${ }^{15} \mathrm{~N}$ NMR relaxation, employing different theoretical formalisms such as spectral density mapping, ${ }^{6-11}$ extended model-free analysis, ${ }^{12}$ continuous distribution of correlation times, $^{13,14}$ and isotropic reorientational eigenmode dynamics (iRED) $)^{15}$ as well as polymer models. ${ }^{16-18}$ Since ${ }^{15} \mathrm{~N}$ relaxation rates in IDPs generally increase only slightly with protein molecular weight, it is believed that backbone reorientation in IDPs is predominantly governed by internal segmental motions independent of the overall tumbling of protein. ${ }^{19}$ More specifically, concerted motions involving local clusters of side chains, entire subdomains, or non-native transient long-range interactions were evidenced in some denatured proteins and proteins in intrinsically disordered states. ${ }^{6,12,14,15,20-28}$ These motions are characterized by the presence of correlation times of several nanoseconds for the dipolar interactions between protein nuclei and are ascribed to the persistence of structural preferences within the ensembles of conformational states. It may thus appear that unfolded proteins are not completely disordered but retain some degree of partial order, so that even peptide segments distant in sequence experience a correlated motion because they temporarily interact with one another. Nevertheless, there is not yet a clear picture of the generality of the presence of concerted motions in IDPs.

High-field relaxation data are not ideally suited to study concerted motions in disordered proteins, because at such fields the small fraction of spectral density function related to the correlated motions with longer correlation times is largely dispersed. On the other hand, fast field cycling (FFC) relaxometry allows measurement of ${ }^{1} \mathrm{H} R_{1}$ values from very low fields up to $50 \mathrm{MHz}$ and provides direct visualization of the

Received: July 7, 2014

Published: October 21, 2014 
spectral density function at low frequencies. Relaxometry measurements can thus clarify and confirm the extent of correlated motions with long correlation times in disordered proteins. The presence of a dispersion at low fields in the relaxation rate profile of protein protons (performed in $\mathrm{D}_{2} \mathrm{O}$ solutions in such a way as to only observe the nonexchangeable protein protons) can in fact directly provide the largest correlation time values that modulate the dipolar interactions between protein protons. ${ }^{29}$ FFC measurements are now feasible, due to the availability of high-sensitivity fast fieldcycling relaxometers, with improved sensitivity with respect to that used in Bertini et al. ${ }^{29}$ The new instrument can directly detect signals of protein protons in millimolar solutions in $\mathrm{D}_{2} \mathrm{O}$ over a very wide field range (from a few kilohertz to tens of megahertz of proton Larmor frequency), being thus able to provide the collective relaxation dispersion profile of protein protons in solution. ${ }^{30}$ This collective relaxation dispersion profile can then be analyzed in terms of an overall "collective" order parameter and an overall rotational correlation time. ${ }^{31}$

Protein proton relaxation measurements in $\mathrm{D}_{2} \mathrm{O}$ were first performed on globular proteins, which are expected to be subject to Brownian motion with a reorientation time $\tau_{\mathrm{R}}$ that depends on the molecular weight (MW) of the protein. The proton-proton dipolar interactions within each protein molecule are modulated by the Brownian motion of the molecule, so that the protein proton relaxation rate profiles must show a dispersion at fields corresponding to the relationship $\omega \tau_{\mathrm{R}}=1$. Indeed, such dispersion has been observed using FFC. ${ }^{29,31,32}$ Surprisingly, measurements performed on the IDP $\alpha$-synuclein also showed a small dispersion at $\omega \tau_{\mathrm{R}}=1$ with a correlation time comparable to the reorientation time calculated for a folded protein with the same molecular weight. ${ }^{29}$ The dispersion should not be observed if $\alpha$ synuclein was only subject to segmental motions with correlation times much shorter than the reorientation time of the protein, ${ }^{16,33}$ since the dispersions corresponding to such times occur at much larger fields. The observed dispersion therefore suggested that a residual correlated motion is present in $\alpha$-synuclein. At the same time, a small Lipari-Szabo $S_{\mathrm{C}}{ }^{2}$ parameter $^{34}$ of $0.05-0.1$ was observed for the collective protein protons, in line with the disordered nature of $\alpha$-synuclein: the collective relaxation rate of protein protons at low fields, which are hundreds or thousands of $\mathrm{s}^{-1}$ in regularly folded proteins, drastically reduces to few tens of $\mathrm{s}^{-1}$ in the case of $\alpha$ synuclein. $^{29,31}$

The observation of a long reorientational time in $\alpha$-synuclein suggests that relaxation measurements of protein protons at low fields provide access to residual correlated motions in IDPs. However, the question remained if long correlation time is unique to $\alpha$-synuclein or if it is a common feature of disordered proteins. To address this question we here performed relaxation measurements for four unfolded proteins of similar MW, some of them known to experience specific intramolecular interactions and some others expected to have much smaller or absent correlated motions. Besides different mutants of $\alpha$ synuclein, we investigated the proteins $\beta$-synuclein, denatured lysozyme, and the K19 fragment (99 amino acids) of the protein Tau. We show that a dispersion of similar amplitude and corresponding to a correlation time of $5-10 \times 10^{-9} \mathrm{~s}$ is present in all the studied proteins. In addition, in case of the protein Tau, which has a molecular weight of $45.8 \mathrm{kDa}$, a second even longer correlation time was detected. Moreover, and in support for the generality of these slow motions in IDPs, we show by ensemble-based hydrodynamic calculations that the time scale of reorientational motion in IDPs can be satisfactorily reproduced on the basis of friction-mediated coupling between their statistical segments. Our work thus provides insight into the fundamental physicochemical properties of IDPs and indicates that all disordered polypeptides made by natural amino acids move in water in a more correlated way than previously expected.

\section{METHODS}

Protein Sample Preparation. Protein samples were prepared by dissolving about $10 \mathrm{mg}$ of the lyophilized proteins in $0.5 \mathrm{~mL} \mathrm{D}_{2} \mathrm{O}$. Wild-type $\alpha$-synuclein and its mutants as well as $\beta$-synuclein were prepared as previously described. ${ }^{35,36}$ The Tau protein and its K19 fragment were prepared as reported elsewhere. ${ }^{37,38}$

Unfolded hen egg white lysozyme was prepared by dissolving the lyophilized protein from Sigma-Aldrich in $\mathrm{D}_{2} \mathrm{O}$, lyophilizing it again, and dissolving the powder in a deuterated $\mathrm{D}_{2} \mathrm{O}$ buffer containing $6 \mathrm{M}$ guanidinium chloride, $1 \mathrm{mM}$ EDTA, $75 \mathrm{mM}$ DTT, $0.1 \mathrm{M}$ Tris ( $\mathrm{pH}^{*}$ 8.0). The buffer was prepared by lyophilizing a DTT solution in $\mathrm{D}_{2} \mathrm{O}$, dissolving the powder in $\mathrm{D}_{2} \mathrm{O}$, lyophilizing it again, dissolving again the powder in $\mathrm{D}_{2} \mathrm{O}$, and adding to a solution of deuterated guanidinium chloride, EDTA, and Tris. The latter solution was prepared by dissolving $6 \mathrm{M}$ guanidinium chloride, $1 \mathrm{mM}$ EDTA, and $0.1 \mathrm{M}$ Tris in $\mathrm{H}_{2} \mathrm{O}$, drying under reduced pressure, adding $\mathrm{D}_{2} \mathrm{O}$ to the powder, and drying again under reduced pressure. This cycle was repeated two more times, then the powder was placed in a drier with $\mathrm{P}_{2} \mathrm{O}_{5}$, and finally $\mathrm{D}_{2} \mathrm{O}$ (99.996\% isotope purity) was added.

Relaxation Rate Profiles. Magnetization decay/recovery curves were recorded at $298 \mathrm{~K}$ from 0.02 to $45 \mathrm{MHz}$ using a Stelar fast field cycling relaxometer of the latest generation. ${ }^{30}$ The sensitivity of this instrument allows detection of the signal of protein protons at millimolar protein solutions in $\mathrm{D}_{2} \mathrm{O}$ with a good signal-to-noise ratio. ${ }^{31}$ Magnetization decays from a prepolarized intensity were measured for relaxation fields up to $10 \mathrm{MHz}$, using a polarization time of $0.5 \mathrm{~s}(1.5 \mathrm{~s}$ for the N30 $\alpha$-synuclein fragment and the K19 fragment of the Tau protein) and a polarization field of $30 \mathrm{MHz}$. The points in the decay were acquired at 64 time values logarithmically scaled between 0.001 or 0.005 and $0.48 \mathrm{~s}$. At higher fields, magnetization recovery curves were measured through acquisition of the magnetization signal for 64 time values logarithmically scaled between 0.003 and $1.2-4 \mathrm{~s}$. The number of scans was 64 and 32 for the decay (low fields) and recovery (high fields) curves, respectively.

The collective protein proton relaxation rates up to $10 \mathrm{MHz}$ were obtained for each field of measurement from the fit of the measured magnetization decay curves. The latter were subjected to a biexponential $\mathrm{fit}^{31}$ with fixed weights, which resulted in excellent agreement with the experimental data. The weight of the exponential function with the smaller rate was adjusted to 0.55 , similar to the 0.60 value previously estimated from a more complex analysis on folded proteins, ${ }^{31}$ and the fit has been performed by imposing that the ratio $f$ between the largest and the smallest relaxation rates is constant for all the curves detected at any magnetic field. The largest relaxation rate is calculated in all cases to be $f=2.5 \pm 0.5$ times larger than the smallest relaxation rate, in line with relaxation dispersion measurements in regularly folded proteins. ${ }^{31}$

The collective protein proton relaxation rates for fields larger than $10 \mathrm{MHz}$ were obtained from the fit of the magnetization recovery curves using a three-exponential function. This function was written with the first two terms defined in the same way as for the magnetization decay curves at lower fields, while the third term corresponds to the field-independent relaxation of residual water protons present in solution. The water proton longitudinal relaxation rate was directly measured at $400 \mathrm{MHz}$ for $\alpha$-synuclein and was found to be $0.07 \mathrm{~s}^{-1}$. The experimentally determined rate was included as the relaxation rate in the third term of the three-exponential function, and a very good fit was obtained (Figure S1). Subsequently, for consistency a third term was also included in the fit of the low-field magnetization decay curves, resulting in the following functions for fitting: 


$$
M=M_{0}\left(0.55 \exp \left(-R_{1 s} t\right)+0.45 \exp \left(-R_{1 s} f t\right)\right)+M_{\mathrm{w}} \exp (-0.07 t)+k
$$

$$
\begin{aligned}
M= & M_{0}\left(0.55\left(1-\exp \left(-R_{1 s} t\right)\right)+0.45\left(1-\exp \left(-R_{1 \mathrm{~s}} t t\right)\right)\right) \\
& +M_{\mathrm{w}}(1-\exp (-0.07 t)+k
\end{aligned}
$$

for the magnetization decay and recovery curves at low and high magnetic fields, respectively, where $M_{0}, R_{1 s}, f, M_{\mathrm{w}}$, and $k$ are fit parameters, and $f$ was kept constant at all fields. The collective relaxation rate values, corresponding to the weighted average of the relaxation rates of all nonexchangeable protons in the protein, were then calculated as $0.55 R_{1 \mathrm{~s}}+0.45 R_{1 \mathrm{~s}} f$.

High-field relaxation rate measurements were performed at an NMR spectrometer with a proton Larmor frequency of $400 \mathrm{MHz}$ using an inversion recovery pulse sequence. Nonselective $R_{1}$ was obtained with a monoexponential fit of the intensity of the protein $1 \mathrm{D}$ NMR peaks using 13 inversion delays ranging from $1 \mathrm{~ms}$ to $5 \mathrm{~s}$.

Hydrodynamic Calculations. For hydrodynamic calculations, the recently introduced method of "hydrodynamic coupling of domains" (HYCUD) was used. ${ }^{39}$ Here, we combined HYCUD with FlexibleMeccano, which builds random structures on the basis of amino acidspecific conformational propensities derived from a library of coil conformations in high-resolution X-ray structures. ${ }^{40}$ Two ensembles of $\alpha$-synuclein, each containing 5000 random structures, were used for the HYCUD calculations. The two ensembles were generated by the Flexible-Meccano program, with or without introduction of a distance constraint between $\mathrm{N}$ - and C-terminal residues. The long-range contact between the $\mathrm{N}$ - and $\mathrm{C}$-terminal residues of $\alpha$-synuclein was defined as a distance $<15 \AA$ between at least one $C^{\beta}$ atom of residues $1-20$ and any of the $C^{\beta}$ atoms of residues $121-140$. Introduction of this long-range contact has been shown to improve the fit to experimental RDCs of $\alpha$-synuclein. ${ }^{40}$ In addition, ensembles of 5001000 random structures of C-terminal truncated $\alpha$-synuclein $(1-108)$, $\beta$-synuclein (134 residues), full-length Tau protein (1-441), and Tau K19 fragment (99 residues) and 5000 random poly alanine (1-100) models without any long-range distance constraint were generated. For all synuclein, Tau and poly alanine ensembles, side chain heavy atoms were added by the program SSCOMP. HYCUD calculations were performed as described in, ${ }^{39}$ using an in-house Python script. Briefly, following the worm-like chain model, ${ }^{41}$ each member of the protein ensembles was split into nonoverlapping fragments of $\sim 14$ residues length. The chosen length of 14 residues is twice the persistence length estimated for denatured proteins. ${ }^{16,17}$ The program Hydropro ${ }^{42}$ was then used to calculate the initial $\tau_{\mathrm{R}}\left(\tau_{\mathrm{R}, 0}\right)$ and intrinsic viscosity $\left(\left[\eta_{0}\right]\right)$ for each segment. Subsequently, the $\tau_{\mathrm{R}, 0}$ of each segment was corrected by a scaling factor derived from the $\left[\eta_{0}\right]$ of all other segments and the distance of their centers of mass from that of the segment of interest. This correction factor approximates the effective relative viscosity experienced locally by each segment, which slows down its reorientational motion. ${ }^{39}$ The corrected $\tau_{\mathrm{R}}$ of each segment was then averaged over the whole ensemble to represent its $\tau_{\mathrm{R}}$ in the context of full-length protein.

\section{RESULTS}

Protein Proton Relaxation Profiles and Theoretical Framework for Their Analysis. Field cycling relaxometry provides longitudinal relaxation rates of sample nuclei over a wide field range, so that their whole spectral density is experimentally and directly available. Since the intrinsically low sensitivity of this technique, however, provides a single unresolved signal, information on nonexchangeable protein protons can be directly accessed by dissolving the proteins in $\mathrm{D}_{2} \mathrm{O}$.

The collective protein proton relaxation rate values, obtained from the measured magnetization decay/recovery curves (Figure S1) by considering that different pools of protons have different relaxation rates, as analyzed in ref 31 and described in the Methods section, are reported as a function of field in Figure 1 for the proteins $\alpha$-synuclein, $\beta$-synuclein, denatured lysozyme, and the K19 fragment of the Tau protein.

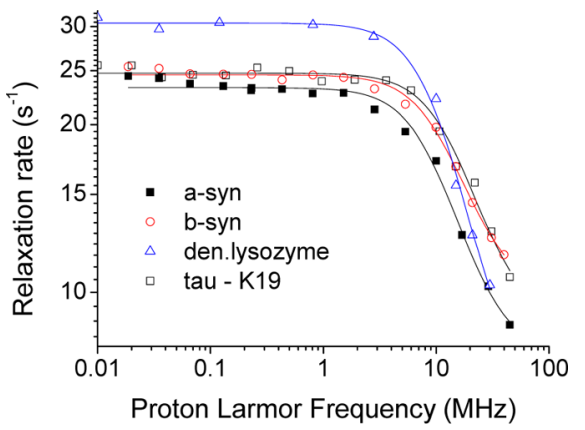

Figure 1. Collective protein proton relaxation rates of $\alpha$-synuclein, $\beta$ synuclein, denatured lysozyme, and the K19 fragment of the protein Tau. Solid lines show the best-fit profiles according to eq 2 .

All relaxation profiles showed one dispersion at approximately $10 \mathrm{MHz}$. In general, such dispersion provides a $\tau_{\mathrm{R}}$ value of the order of the reorientation time that the protein would have if it were folded. ${ }^{29,31}$ The profiles were subsequently fit to eq 2 :

$$
R_{1}=S_{\mathrm{C}}{ }^{2}\left\langle\mathrm{E}^{2}\right\rangle\left(\frac{0.8 \tau_{\mathrm{R}}}{1+4 \omega^{2} \tau_{\mathrm{R}}^{2}}+\frac{0.2 \tau_{\mathrm{R}}}{1+\omega^{2} \tau_{\mathrm{R}}^{2}}\right)+\alpha
$$

where $\tau_{\mathrm{R}}, S_{\mathrm{C}}{ }^{2}$, and $\alpha$ are fit parameters, and $R_{1}$ is reported on a logarithmic scale (Figure 1). $\left\langle E^{2}\right\rangle$ represents a measure of the mean squared energy related to all proton-proton dipolar interactions. In folded proteins, $\left\langle E^{2}\right\rangle$ was found to be fairly constant and equal to $(27 \pm 3) \times 10^{9} \mathrm{~s}^{-2}$. ${ }^{31}$ In the case of unfolded proteins, the value is expected to be slightly smaller because of loosening of dipolar interactions between protons belonging to different residues, whereas the intramethylene and intramethyl proton-proton dipolar interactions are maintained. In this study, $\left\langle E^{2}\right\rangle$ was estimated using the program CORMA ${ }^{43}$ and the structure of micelle-bound $\alpha$-synuclein (PDB code: 1XQ8) and gave a value of $24 \times 10^{9} \mathrm{~s}^{-2} \cdot{ }^{44}$ Similar values $( \pm 1 \times$ $10^{9} \mathrm{~s}^{-2}$ ) were obtained using minimized polypeptide chain structures of totally unfolded $\alpha$-synuclein. Therefore, in all fits $\left\langle E^{2}\right\rangle$ was fixed to this value.

The best-fit values of $\tau_{\mathrm{R}}, S_{\mathrm{C}}{ }^{2}$, and $\alpha$ for the different proteins are reported in Table 1 . In all cases, $S_{\mathrm{C}}{ }^{2}$ values of about 0.1 were obtained. This parameter is defined as a squared collective order parameter which reflects the presence of global motions with long correlation time. The latter is found to be in the range $(6-9) \times 10^{-9} \mathrm{~s}$, i.e., only slightly smaller than the value expected for a rigid protein of MW 15,000 in $\mathrm{D}_{2} \mathrm{O}$ at $25^{\circ} \mathrm{C}$. In the case of folded lysozyme $\left(\mathrm{pH}^{*} 3.5\right)$, for instance, $\tau_{\mathrm{R}}$ was calculated and experimentally found through relaxation measurements to be $9.0 \times 10^{-9} \mathrm{~s}^{31}$ Therefore, the relaxation dispersion measurements indicate that unfolded proteins share the general feature of having a minor component of this motion which is of the order of the reorientational time of folded proteins of similar MW. No difference in the relaxation profile was observed for the $\alpha$-synuclein sample when the concentration of the protein was decreased from 1.5 to $0.75 \mathrm{mM}$, excluding significant contributions from transient intermolecular interactions.

Protein Proton Relaxation Rates at High Fields. Protein proton relaxation rates were measured for the same proteins at $400 \mathrm{MHz}$. The nonselective $R_{1}$ values for different peaks in the ${ }^{1} \mathrm{H}$ NMR spectrum were determined (ranging from 0.6 to 2.4 
Table 1. Best-Fit Parameters Obtained from the Relaxation Dispersion Profiles of Protein Protons

\begin{tabular}{|c|c|c|c|c|}
\hline & $\alpha$-synuclein (140 AA) & $\beta$-synuclein (134 AA) & denatured lysozyme (129 AA) & Tau K19 fragment (99 AA) \\
\hline$\tau_{\mathrm{R}}(\mathrm{ns})$ & $7.9 \pm 0.5$ & $6.5 \pm 0.3$ & $6.1 \pm 0.9$ & $5.3 \pm 0.4$ \\
\hline$S_{\mathrm{C}}^{2}$ & $0.08 \pm 0.01$ & $0.09 \pm 0.01$ & $0.14 \pm 0.02$ & $0.12 \pm 0.01$ \\
\hline$\alpha\left(\mathrm{s}^{-1}\right)$ & $7.7 \pm 0.3$ & $9.9 \pm 0.3$ & $7.2 \pm 0.6$ & $8.7 \pm 0.6$ \\
\hline$\tau_{\mathrm{R}}(\mathrm{ns})$ in water ${ }^{a}$ & $6.3 \pm 0.4$ & $5.2 \pm 0.3$ & $4.9 \pm 0.7$ & $4.2 \pm 0.3$ \\
\hline HYCUD-predicted $\tau_{\mathrm{R}}(\mathrm{ns})^{b}$ & $5.2(4.5-6.7)$ & $5.0(4.1-6.2)$ & - & $4.9(4.2-5.9)$ \\
\hline
\end{tabular}

${ }^{a}$ Corrected for the difference in viscosity between pure $\mathrm{D}_{2} \mathrm{O}$ (used in proton relaxation measurements) and $\mathrm{H}_{2} \mathrm{O}$ (assumed in HYCUD calculations). ${ }^{b}$ The HYCUD-predicted $\tau_{\mathrm{R}}$ was calculated with an AER value of $2.9 \AA \AA$. The values in parentheses report the range of predicted $\tau_{\mathrm{R}}$, when AER varied between 2.6 and $3.3 \AA$.

$\mathrm{s}^{-1}$ ) and then averaged after weighting according to the area of the peak. All samples provided averaged relaxation rate values in the range of $1.8-1.9 \mathrm{~s}^{-1}$. These values are smaller than the limiting $\alpha$ values obtained from the fit of the relaxation profiles at low fields (Table 1). This indicates that the relaxation rates undergo a further sizable decrease between the highest fields available for relaxometry $(45 \mathrm{MHz})$ and $400 \mathrm{MHz}$, and therefore further dispersions with times smaller than the calculated $\tau_{\mathrm{R}}$ and larger than approximately $0.2 \times 10^{-9} \mathrm{~s}$ must be present (see the Discussion section).

Protein Proton Relaxation Profiles of $\alpha$-Synuclein Variants. In the case of $\alpha$-synuclein, transient secondary structures present in the wild-type protein are partially perturbed by the genetic variants A53T and A30P. ${ }^{35,40,45,46}$ We therefore probed the effect of these mutations on protein proton relaxation profiles. In addition, we analyzed the protein proton relaxation profiles of the $\alpha$-synuclein mutant V118A/ $\mathrm{M} 127 \mathrm{~A}$, in which two hydrophobic residues in the C-terminal tail were replaced by alanine and of a C-terminally truncated variant (residues 1-108). In all cases (Figure 2), a low-field

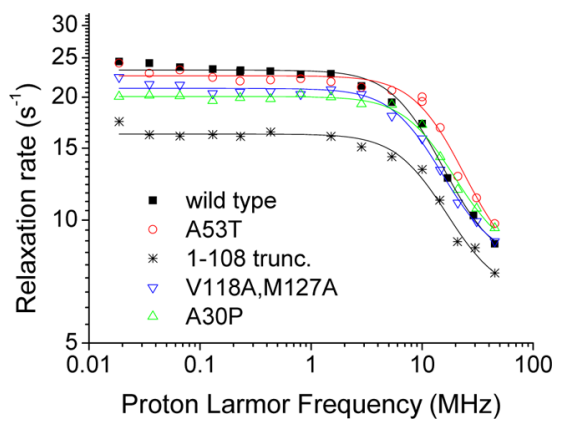

Figure 2. Collective protein proton relaxation rates of different $\alpha$ synuclein variants. The profile observed for wild-type $\alpha$-synuclein is reported for comparison. Solid lines show the best-fit profiles obtained according to eq 2 . dispersion was present with a correlation time of $(5-8) \times 10^{-9}$ $\mathrm{s}$ (Table 2) and the $S_{\mathrm{C}}{ }^{2}$ values remained close to the value obtained for wild-type $\alpha$-synuclein. In line with a perturbation of the ensemble of conformers by the mutations and the corresponding partial disruption of transient local and longrange contacts, the correlation time of A53T and A30P $\alpha$ synuclein decreased when compared to the wild-type protein, while the control mutant V118, M127A was indistinguishable (Table 2): dispersions for the A53T and A30P proteins, in fact, occur at larger fields than that of the wild-type protein. This resulted in relaxation rates of the mutants that are smaller at low fields (only slightly in the case of the A53T mutant due to the increased $S_{\mathrm{C}}{ }^{2}$ value) and larger at high fields $(20-40 \mathrm{MHz})$ than those of the wild-type $\alpha$-synuclein. In addition, the $\mathrm{C}$ terminal truncated variant showed a decreased $\tau_{\mathrm{R}}$ value, consistent with its smaller MW.

Protein Proton Relaxation Profiles of IDPs of Different Chain Length. To test the influence of increasing chain length on protein proton correlation times, we performed additional measurements for a peptide comprising the N-terminal 30 residues of $\alpha$-synuclein (N30 $\alpha$-synuclein) as well as htau40, the longest isoform of Tau in the human brain with 441 residues (Figure 3 ). In the case of the N30 $\alpha$-synuclein, the low-field relaxation rate is halved with respect to the value of the wild-type 140-residue protein, and the dispersion is only seen starting at the largest magnetic fields. In this way, an upper limit to $\tau_{\mathrm{R}}$ can only be estimated, around $2 \times 10^{-9} \mathrm{~s}$.

The low-field relaxation rate of 441-residue htau40 is roughly doubled with respect to the values of wild-type $\alpha$-synuclein and the 99-residue Tau fragment K19. Furthermore, the dispersion is moved toward lower fields, pointing to a larger correlation time. A fit with a single $\tau_{\mathrm{R}}$ was attempted (dotted line in Figure 3 ), providing a value of $10 \times 10^{-9} \mathrm{~s}$ with $S_{\mathrm{C}}{ }^{2}=0.12$. Both the calculated squared order parameters and $\tau_{\mathrm{R}}$ are thus larger than in $\alpha$-synuclein, and the product of the two values is about doubled when compared to $\alpha$-synuclein. In addition, the dispersion is moved toward lower fields when compared to the profile of $\mathrm{K} 19$, pointing to a doubling in $\tau_{\mathrm{R}}$ when the same squared order parameter is assumed. However, Figure 3

Table 2. Best-Fit Parameters Obtained from the Proton Relaxation Dispersion Profiles of $\alpha$-Synuclein and Its Variants

\begin{tabular}{|c|c|c|c|c|c|}
\hline & wild-type & A53T & A30P & V118A, M127A & $1-108$ (truncated) \\
\hline$\tau_{\mathrm{R}}(\mathrm{ns})$ & $7.9 \pm 0.5$ & $5.0 \pm 0.5$ & $5.8 \pm 0.3$ & $7.7 \pm 0.5$ & $6.9 \pm 0.7$ \\
\hline$S_{\mathrm{C}}^{2}$ & $0.08 \pm 0.01$ & $0.13 \pm 0.01$ & $0.08 \pm 0.01$ & $0.07 \pm 0.01$ & $0.06 \pm 0.01$ \\
\hline$\alpha\left(\mathrm{s}^{-1}\right)$ & $7.7 \pm 0.3$ & $7.2 \pm 0.7$ & $8.0 \pm 0.3$ & $7.9 \pm 0.3$ & $6.5 \pm 0.4$ \\
\hline$\tau_{\mathrm{R}}(\mathrm{ns})$ in water ${ }^{a}$ & $6.3 \pm 0.4$ & $4.0 \pm 0.4$ & $4.6 \pm 0.3$ & $6.2 \pm 0.4$ & $5.5 \pm 0.6$ \\
\hline HYCUD-predicted $\tau_{\mathrm{R}}(\mathrm{ns})^{b}$ & $5.2(4.5-6.7)$ & $5.3(4.4-6.3)$ & $5.2(4.4-6.3)$ & $5.3(4.5-6.6)$ & $4.5(3.8-5.5)$ \\
\hline
\end{tabular}

${ }^{a}$ Corrected for the difference in viscosity between pure $\mathrm{D}_{2} \mathrm{O}$ (used in proton relaxation measurements) and $\mathrm{H}_{2} \mathrm{O}$ (assumed in HYCUD calculations).

${ }^{b}$ The HYCUD-predicted $\tau_{\mathrm{R}}$ were calculated with an AER value of $2.9 \AA$. The values in parentheses report the range of predicted $\tau_{\mathrm{R}}$, when AER varied between 2.6 and $3.3 \AA$. 


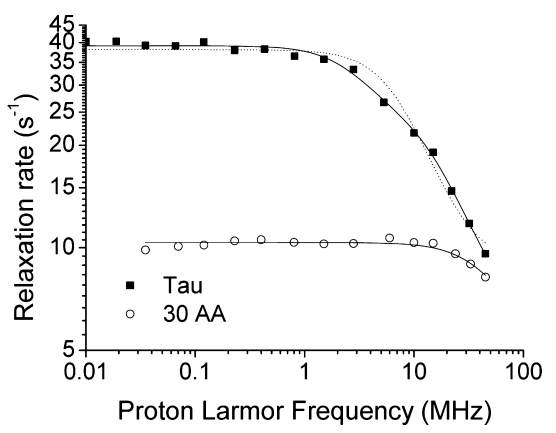

Figure 3. Dependence of protein proton relaxation rates on chain length. Collective protein proton relaxation rates of a peptide comprising the $\mathrm{N}$-terminal 30 residues of the protein $\alpha$-synuclein (open circles) and of 441-residue Tau protein (black squares) are shown. The fit with a single correlation time for the protein Tau is shown as dotted line, and the fit with two correlation times (eq 3 ) as solid line.

indicates that the fit is not optimal. It was thus repeated by including two correlation times, according to the equation: ${ }^{32}$

$$
\begin{aligned}
R_{1} & =\left\langle E^{2}\right\rangle\left[S_{\mathrm{C}(1)}^{2}\left(\frac{0.8 \tau_{\mathrm{R} 1}}{1+4 \omega^{2} \tau_{\mathrm{R} 1}^{2}}+\frac{0.2 \tau_{\mathrm{R} 1}}{1+\omega^{2} \tau_{\mathrm{R} 1}^{2}}\right)\right. \\
& \left.+S_{\mathrm{C}(2)}{ }^{2}\left(\frac{0.8 \tau_{\mathrm{R} 2}}{1+4 \omega^{2} \tau_{\mathrm{R} 2}{ }^{2}}+\frac{0.2 \tau_{\mathrm{R} 2}}{1+\omega^{2} \tau_{\mathrm{R} 2}{ }^{2}}\right)\right]+\alpha
\end{aligned}
$$

The fit using two correlation times $\tau_{\mathrm{R} 1}$ and $\tau_{\mathrm{R} 2}$ (solid line in Figure 3) resulted in $\tau_{\mathrm{R} 1}=(27 \pm 0.5) \times 10^{-9} \mathrm{~s}, S_{\mathrm{C}(1)}{ }^{2}=0.02$ and $\tau_{\mathrm{R} 2}=4 \times 10^{-9} \mathrm{~s}, S_{\mathrm{C}(2)}{ }^{2}=0.17$ with $\alpha=5.9 \mathrm{~s}^{-1}$ (Table S1). Although the increased number of fit parameters may introduce overfitting of the experimental data, we note that the largest correlation time obtained from this fit has a similar magnitude as the reorientational time expected for a rigid protein of MW $45,850\left(28 \times 10^{-9} \mathrm{~s}\right)$, i.e., about 3 times larger than that of lysozyme. The $S_{\mathrm{C}}{ }^{2}$ corresponding to this largest correlation time decreases to 0.02 .

The measurements indicate that the position of the dispersion depends on the MW of the protein, and the corresponding correlation time increases with chain length. The protein proton relaxation rates of htau 40 also suggest that the observed dispersion is not likely to correspond to a unique Lorentzian dispersion but rather to a superposition of dispersions with different $\tau_{\mathrm{R} i}$ values up to a maximum value. Notably, the nonselective $R_{1}$ measurements performed at 400 $\mathrm{MHz}$ gave an averaged protein proton relaxation rate of $1.7 \mathrm{~s}^{-1}$ for htau40, a value similar to those observed for smaller proteins.

Relaxation rate measurements performed at $400 \mathrm{MHz}$ as well as the relaxation profile obtained for the 441-residue Tau protein htau40 indicate that the spectral density is not a single Lorentzian. We checked that it is indeed possible to reproduce the field dependence of the protein proton relaxation rate of $\alpha$ synuclein, htau 40 , and of the N30 $\alpha$-synuclein peptide using eq $4:^{47}$

$$
\mathrm{R}_{1}=\sum_{i}\left\langle E^{2}\right\rangle\left[\mathrm{S}_{\mathrm{C} i}^{2}\left(\frac{0.8 \tau_{\mathrm{R} i}}{1+4 \omega^{2} \tau_{\mathrm{R} i}^{2}}+\frac{0.2 \tau_{\mathrm{R} i}}{1+\omega^{2} \tau_{\mathrm{R} i}^{2}}\right)\right]
$$

and 3-5 correlation times $\tau_{\mathrm{R} i}$ with the constraints that the sum of $S_{\mathrm{C} i}{ }^{2}$ is $1, \tau_{\mathrm{R} 1}$ is equal to the reorientation time of a rigid protein with the same MW and $\tau_{\mathrm{R}(i+1)} / \tau_{\mathrm{R} i}$ is 0.2 (Figure S2).
Prediction of $\alpha$-Synuclein Reorientational Correlation Time by HYCUD. Disordered proteins exhibit a persistence length of 7 residues, ${ }^{16,17}$ and the $\tau_{\mathrm{R}}$ of 14 -residue statistical (Kuhn's) segments of $\alpha$-synuclein at $25{ }^{\circ} \mathrm{C}$ lies in the range of $(1.3-1.6) \times 10^{-9} \mathrm{~s}$, much shorter than the experimentally observed $\tau_{\mathrm{R}}$ (Figure 4 ). The long $\tau_{\mathrm{R}}$ of $\alpha$-synuclein therefore

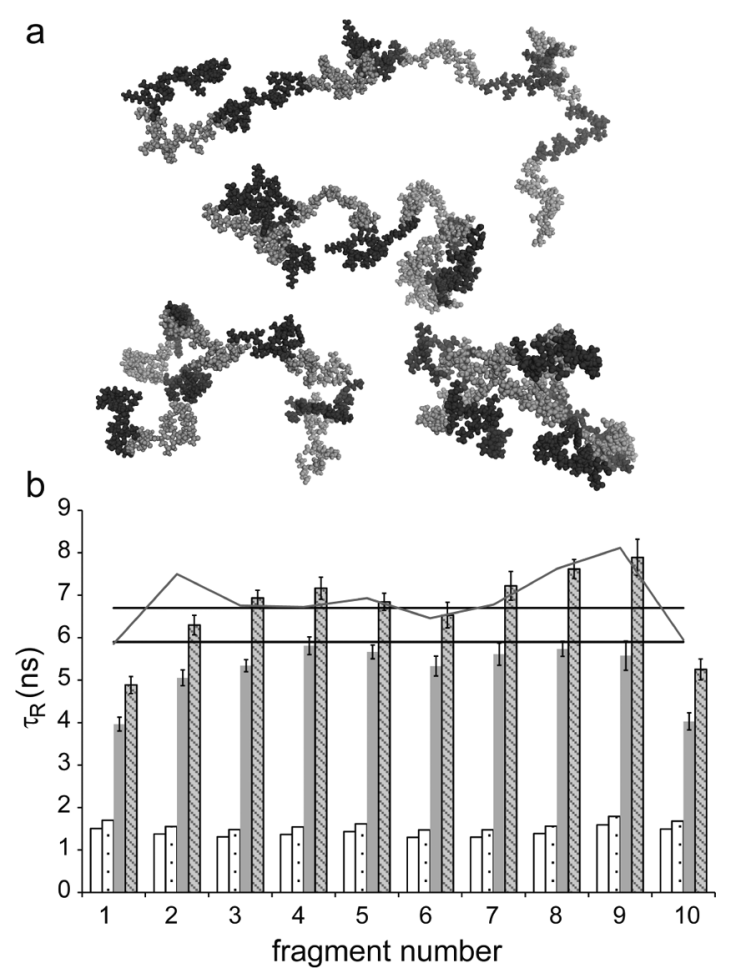

Figure 4. HYCUD predictions of effective reorientational correlation time $\left(\tau_{\mathrm{R}}\right)$ in $\alpha$-synuclein. (a) Four representative models of the structural ensemble of $\alpha$-synuclein used in HYCUD calculations. The 10 fragments of $\alpha$-synuclein each containing 14 residues are depicted with alternate dark and light colors. (b) The ensemble-averaged $\tau_{\mathrm{R}}$ calculated for fragments in isolation (white column: AER $2.9 \AA$; white column with dots: AER $3.3 \AA$ ) or in the context of the whole $\alpha$ synuclein molecule (gray: AER 2.9 Å; hatched-column: AER $3.3 \AA$ ). The gray solid line shows the HYCUD-predicted $\tau_{\mathrm{R}}$ of fragments in the context of the whole $\alpha$-synuclein molecule, averaged over a second ensemble of $\alpha$-synuclein in which all conformers contain a long-range contact between the $\mathrm{N}$ - and C-terminus (AER $2.9 \AA$ ). The averages \pm standard deviation of the global $\tau_{\mathrm{R}}$ derived from proton relaxation data (after correction for the viscosity difference) are shown as solid black lines.

points to the long-range correlated dynamics of multiple protein segments due to their mutual interactions. A molecular basis for the observed rotational correlation time might be friction-mediated hydrodynamic coupling of different segments of $\alpha$-synuclein. To test this hypothesis, the method of HYCUD was used. ${ }^{39}$ HYCUD was previously shown to capture the deceleration of reorientational movement of individual domains in flexible multidomain proteins. ${ }^{39,48-51}$

For $\alpha$-synuclein, the $\tau_{\mathrm{R}}$ prediction converged within $500 \alpha$ synuclein conformers (Figure S3) and resulted in a HYCUDpredicted average $\tau_{\mathrm{R}}$ of $(5.2 \pm 0.7) \times 10^{-9} \mathrm{~s}$. The bell-shaped profile of HYCUD-predicted $\tau_{\mathrm{R}}$ along the $\alpha$-synuclein sequence is reminiscent to the profile of NMR residual dipolar couplings (RDC) expected for unfolded proteins (Figure 4). ${ }^{52}$ Next, we explored the influence of simulation parameters on the $\tau_{\mathrm{R}}$ 
prediction of $\alpha$-synuclein. To this end, we changed the atomic effective radius (AER) in the hydrodynamic calculations from $2.9 \AA$, as suggested for globular proteins, ${ }^{42}$ to $3.3 \AA$. While AER of $2.9 \AA$ optimizes the accuracy of predictions for a large set of studied globular proteins, a significant variability has been observed for the AER values (ranged between 2.5 and $3.5 \AA$ ) best predicting hydrodynamic properties of individual proteins. ${ }^{53}$ The larger AER might be more suited for IDPs, as they are more hydrated than a typical folded protein and there is a stronger coupling of their motions with their hydration-layer water molecules. $^{54,55}$ With an AER of $3.3 \AA$, the HYCUDpredicted $\tau_{\mathrm{R}}$ of $\alpha$-synuclein divided into 14-residue fragments increased to $(6.7 \pm 1.0) \times 10^{-9} \mathrm{~s}$ (Figure $4 \mathrm{~b}$ ), in close agreement with the experimental $\tau_{\mathrm{R}}$ (after correction for viscosity difference between $\mathrm{D}_{2} \mathrm{O}$ used in proton relaxometry measurements and $\mathrm{H}_{2} \mathrm{O}$ in HYCUD calculations). Similarly, the $\tau_{\mathrm{R}}$ values of V118A, M127A $\alpha$-synuclein and the truncated variant $(1-108)$ were better reproduced by HYCUD when an AER value of $3.3 \AA$ was used (Table 2 ). On the other hand, close agreements were obtained for $\beta$-synuclein with the AER of $2.9 \AA$, while A30P and in particular A53T variants of $\alpha$ synuclein required smaller AER values (Tables 1 and 2). Since protein sequence and therefore hydration properties are unlikely to be strongly different among synuclein variants, the observed variation in $\tau_{\mathrm{R}}$ might be due to different local and long-range structural propensities.

The $\alpha$-synuclein populates transient long-range interactions between the $\mathrm{N}$ - and $\mathrm{C}$-terminal regions. ${ }^{35,46}$ To investigate their impact on the reorientational dynamics, we generated an ensemble of 5000 conformers of $\alpha$-synuclein with all of them containing at least one contact between residues $1-20$ and $121-40 .^{56}$ In the presence of the long-range contact, the average HYCUD-predicted $\tau_{\mathrm{R}}$ increased to $(6.9 \pm 0.7) \times 10^{-9} \mathrm{~s}$ (Figure $4 \mathrm{~b}$ ). The increase in $\tau_{\mathrm{R}}$ was particularly pronounced for the $\mathrm{N}$ - and $\mathrm{C}$-terminal fragments, i.e., the regions that contained the long-range contact, suggesting that the effective $\tau_{\mathrm{R}}$ of protein fragments calculated by HYCUD for the coil ensemble could potentially serve as a reference to detect and identify protein tertiary contacts. Long-range contacts in $\alpha$ synuclein are probably not limited to the interaction between the $\mathrm{N}$ - and C-terminal regions, as the $\tau_{\mathrm{R}}$ of the $\mathrm{C}$-terminal truncated $\alpha$-synuclein requires a large AER value to be reproduced.

Together, the HYCUD results indicate that the long $\tau_{\mathrm{R}}$ of synuclein variants can be satisfactorily reproduced by hydrodynamic coupling between its rigid statistical segments, comparable with globular proteins, and the accuracy could be particularly improved if the effect of experimentally known long-range contacts is further considered.

HYCUD Prediction of Chain Length Dependence of Reorientational Correlation Time. To investigate if hydrodynamic coupling-mediated rotational deceleration is a general property of disordered polypeptide chains - as suggested by the low-field relaxation dispersion measurements collected for several IDPs - we studied an ensemble of 5000 random structures of a 100-residue poly alanine chain. The results of the HYCUD calculations for this random ensemble (Figure S4) showed a high similarity to the case of $\alpha$-synuclein: the average HYCUD-predicted $\tau_{\mathrm{R}}$ of protein fragments rose from $(0.98 \pm$ $0.04) \times 10^{-9} \mathrm{~s}$ of the isolated state to $(3.35 \pm 0.38) \times 10^{-9} \mathrm{~s}$ in the 100 -residue chain. In addition, the predicted $\tau_{\mathrm{R}}$ increased with chain length (Figure 5a). Thus, we attempted to predict the $\tau_{\mathrm{R}}$ of Tau K19 fragment (99 residues) and a much longer
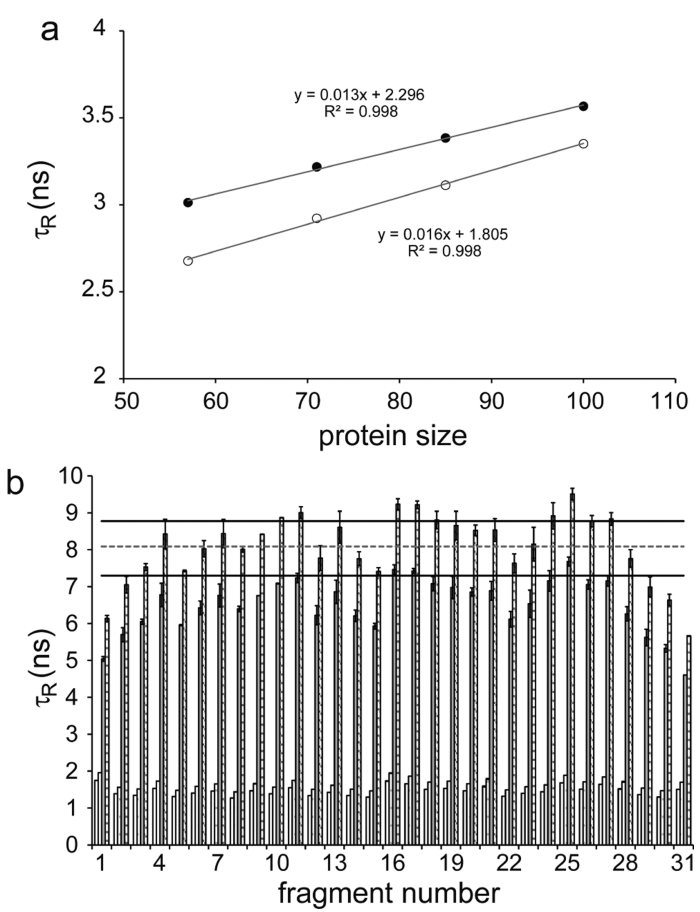

Figure 5. Protein size dependence of reorientational correlation time, $\tau_{\mathrm{R}}$. (a) HYCUD-predicted $\tau_{\mathrm{R}}$ calculated for an ensemble of 5000 random poly alanine structures as a function of protein size (i.e., 56, 70,85 , and 100 residues). Open/filled circles represent average $\tau_{\mathrm{R}}$ values of 14-residue fragments including/excluding the $\mathrm{N}$ - and $\mathrm{C}$ terminal end fragments. (b) The ensemble-averaged $\tau_{\mathrm{R}}$, calculated for fragments in isolation (white column: AER 2.9 A; white column with dots: AER $3.3 \AA$ ) or in the context of the full-length Tau protein (gray: AER $2.9 \AA$ A; hatched-column: AER $3.3 \AA$ ). The gray dotted line shows the HYCUD-predicted $\tau_{\mathrm{R}}$ averaged over fragments in the context of the whole Tau molecule (AER $3.3 \AA$ ). The averages \pm standard deviation of the global $\tau_{\mathrm{R}}$ derived from proton relaxation data (after correction for the viscosity difference) are shown as solid black lines.

IDP, full length tau protein (441 residues) using HYCUD. The $\tau_{\mathrm{R}}$ of Tau K19 fragment (99 residues) is well reproduced with the HYCUD using an AER of $2.6 \AA$ (Table 1 ). The average $\tau_{\mathrm{R}}$ of 14-residue-long fragments of full-length tau protein was predicted at $(8.1 \pm 0.9) \times 10^{-9} \mathrm{~s}$ (with AER $3.3 \AA$, Figure $5 \mathrm{~b}$ ) well agreed to the value of $(7.8 \pm 0.7) \times 10^{-9}$ s suggested by the fit of low-field relaxation dispersion data with a single $\tau_{\mathrm{R}}$ in $\mathrm{H}_{2} \mathrm{O}$. Similar to the case of synuclein, the fact that a larger AER value of $3.3 \AA$ is required to account for the long $\tau_{R}$ of fulllength Tau protein may be originated from the presence of an intricate network of transient long-range contacts in this protein, as demonstrated by NMR data. ${ }^{57}$

Temperature Dependence of Protein Proton Relaxation Rates. To investigate the effect of temperature on protein proton relaxation rates, we performed measurements at 288, 298, and $308 \mathrm{~K}$ for the Tau fragment K19 (Figure 6). With decreasing temperature the relaxation rate values at low fields increased, indicating that the correlation time $\tau_{\mathrm{R}}$ and/or $S_{\mathrm{C}}{ }^{2}$ increases with decreasing temperature. The position of the dispersion indeed shows that $\tau_{\mathrm{R}}$ increases from $(4.6 \pm 0.6) \times$ $10^{-9} \mathrm{~s}$ to $(5.3 \pm 0.4) \times 10^{-9} \mathrm{~s}$ and $(6.6 \pm 0.7) \times 10^{-9} \mathrm{~s}$ when the temperature is lowered from 308 to 298 and $288 \mathrm{~K}$, respectively. The observed changes are in good agreement with the $\eta / T$ dependence of the correlation time (Figure $6 \mathrm{~b}$ ) as expected from Stokes' law (from which values of 4.1 and $6.9 \times$ $10^{-9} \mathrm{~s}$ at 308 and $288 \mathrm{~K}$, respectively, can be calculated using 

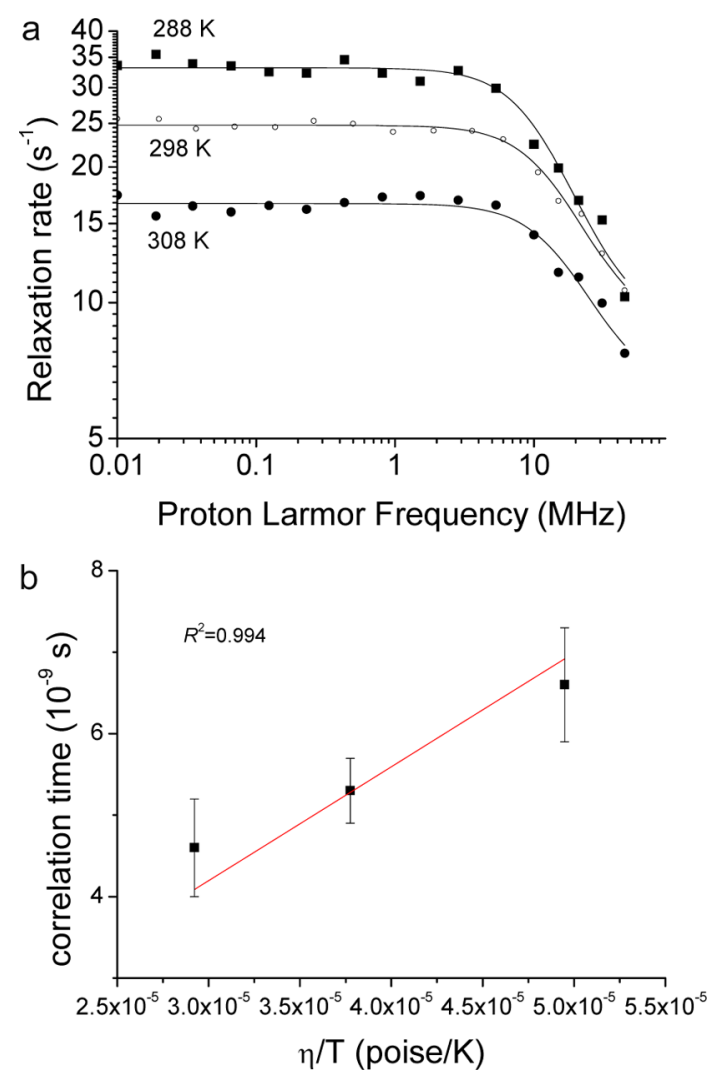

Figure 6. Temperature dependence of protein proton relaxation rates. (a) Collective protein proton relaxation rates observed for the 99residue K19 fragment of the protein Tau at three different temperatures and best-fit profiles. (b) Correlation time as a function of the ratio of viscosity and temperature, $\eta / T$.

$5.3 \times 10^{-9}$ as the correlation time at $\left.298 \mathrm{~K}\right)$. The corresponding $S_{\mathrm{C}}{ }^{2}$ provided by the fit at 308 and $288 \mathrm{~K}$ was $0.09 \pm 0.02$ and $0.15 \pm 0.02$, respectively. We note that $S_{\mathrm{C}}{ }^{2}$ slightly increases with decreasing temperature, passing from 0.09 to 0.12 to 0.15 for temperatures of 308,298 , and $288 \mathrm{~K}$, respectively. Some covariance of $S_{\mathrm{C}}{ }^{2}$ with the $\tau_{\mathrm{R}}$ best-fit value may actually reduce the temperature dependence of $S_{\mathrm{C}}{ }^{2}$ but not abolish it completely.

${ }^{1} \mathrm{H},{ }^{1} \mathrm{H}$ NOEs in C-Terminally Truncated $\boldsymbol{\alpha}$-Synuclein. The sign of proton-proton NOEs depends on the magnitude of the reorientational correlation time. Thus, the presence of negative NOEs (same sign of cross peaks and diagonal peaks) is an indication of slow tumbling. In contrast, positive NOEs indicate that no contributions arise from long correlation times. The NOE intensities can be calculated using eq S1. Figure S5 shows that a correlation time of $6 \mathrm{~ns}$ must be considered with a squared order parameter $S^{2}$ of at least 0.05 to observe negative NOEs at $400 \mathrm{MHz}$, when fast motions are modeled with an average correlation time of 0.4 ns. If only fast motions are present, with no contributions from the overall reorientation time $\left(S^{2}=0\right)$, NOE cross peaks should be positive at $400 \mathrm{MHz}$, unless local motions have correlation times larger than $0.5 \mathrm{~ns}$ (Figure S6). On the other hand, if an overall correlation time of $6 \mathrm{~ns}$ with $S^{2}=0.06$ is assumed, in line with the relaxation measurements (Table 1), fast internal motions of hundreds of picoseconds must be present to obtain negative NOE intensities at $400 \mathrm{MHz}$ (Figure S6). In order to monitor the presence of long correlation times from NOE intensities, 2D NOESY spectra of the C-terminally truncated $\alpha$-synuclein variant (residues $1-108$ ) were acquired at 400 and $700 \mathrm{MHz}$ (Figures S7 and S8). The $400 \mathrm{MHz}$ spectrum indeed showed only negative cross peaks (same sign as the diagonal peaks) with the exception of two cross peaks which presumably involve side chain protons. In the $700 \mathrm{MHz}$ NOESY spectrum, one of the two positive cross peaks became negative. The presence of negative cross peaks in the NOESY spectra-in full agreement with the relaxation measurement results - sets a lower limit for the overall correlation time to about $0.5 \mathrm{~ns}$, in the unrealistic assumption of no faster local motions (as described above and shown in Figure S6). In fact, contributions from spectral density functions characterized by a short correlation time increase the values of the NOEs. Since fast local motions must be present, an overall correlation time on the order of $1 \mathrm{~ns}$ or longer is required.

\section{DISCUSSION}

We have shown that low-field protein proton relaxation rates of a variety of IDPs are much larger than their high-field rates, although much smaller than the rates measured for folded proteins of the same MW (Figure S9). This dispersion from the low-field to the high-field values corresponds to a correlation time of several nanoseconds. This observation demonstrates the presence of a small fraction of long-range correlated motions in IDPs.

Several disordered proteins have been shown to be best described by ensembles of rapidly interconverting conformers. In addition, transient secondary structure and long-range contacts have been identified in many cases, despite the fact that individual ensemble members differ substantially., $36,21,33,58$ The presence of long-range interactions has been determined in particular for denatured lysozyme, ${ }^{12}$ and shown to link clusters of residual secondary structures, ${ }^{17,33}$ and for $\alpha$-synuclein, where they link the $\mathrm{N}$ - and C-terminal regions. ${ }^{35,40,45,46}$ In addition, A53T and A30P mutants of $\alpha$-synuclein have been shown to disrupt residual local structures and enhance local preferences for extended conformations, ${ }^{45}$ although long-range interactions are not completely abolished. ${ }^{59}$ Furthermore, $\beta$-synuclein has been shown to adopt extended conformations characterized by the lack of long-range contacts. ${ }^{19,60}$ The Tau protein also contains regions of residual secondary structure, in particular in the repeat domain and at the C-terminus, ${ }^{38,61,62}$ as well as longrange interactions between its different domains. ${ }^{57}$

High-field ${ }^{15} \mathrm{~N}$ relaxation measurements on unfolded proteins could be satisfactorily explained by the simple model of internal segmental motions, ${ }^{16,19,45}$ especially considering that the relaxation rates (and thus the correlation times) increase only slightly for sizable increase of the protein MW. This has been used to conclude that protein motion is largely independent of the overall tumbling rate. In some cases, as already seen, concerted motions ascribed to transient long-range interaction causing structural preferences have been invoked. ${ }^{6}$ The present measurements shed light on minor but significant components of the motion correlated to reorientations occurring on the several nanosecond time scale, in the absence of specific interactions between protein residues. This has been made possible because the low-field measurements provide the field dependence of the spectral density function at fields for which the dispersion related to such motion can be observed, differently from the high-field measurements, where it is already largely dispersed and only the major, faster segmental motion components remain. 
The ensemble-based hydrodynamic calculations reveal that the time scale of the underlying correlated motions in IDPs are reasonably explained on the basis of hydrodynamic coupling between their statistical fragments. Factors such as presence of temporary tertiary contacts or variation in the hydration level slightly modulate $\tau_{\mathrm{R}}$, but even in their absence, the $\tau_{\mathrm{R}}$ remains larger than what is often captured by the high-field ${ }^{15} \mathrm{~N}$ relaxation data. The presence of correlated motions is also supported by molecular dynamics (MD) simulations performed by Xue and Skrynnikov for the denatured protein ubiquitin. ${ }^{63}$ In fact, the calculations indicated the presence of three exponential components in the correlation functions of the ${ }^{1} \mathrm{H}-{ }^{15} \mathrm{~N}$ vectors, corresponding to correlation times of 44 ps and 1.4 and $9.4 \mathrm{~ns}$, with weights of $0.30,0.42$, and 0.28 , respectively. The latter two components were attributed to local and global conformational transitions. Thus, these calculations show that, although most of the conformational dynamics occurs locally (in the sense that a change in the backbone dihedral angle of a certain residue usually does not cause any global conformational rearrangement), fast local motions are unable of averaging dipolar interactions completely. As a result, a relatively long correlation time of about $10 \mathrm{~ns}$ appeared from the simulations responsible for the long tails of the correlation functions. This long correlation time was ascribed to large-scale conformational rearrangements of the polypeptide chain, i.e., to large displacements of big fragments of the chain. Despite the empirical approach of the modeling, this $\mathrm{MD}$ simulation is thus consistent with the current study and supports the idea that the presence of a long correlation time is an intrinsic property of incomplete averaging of dipole-dipole interactions by segmental motions.

Different types of motion in IDPs such as subnanosecond internal motions, segmental motions, and overall tumbling can be coupled provided that they occur on comparable time scales. ${ }^{64}$ On the other hand, these motions, which are subject to kinetic barriers of different types, exhibit different time scale sensitivity to environmental factors such as temperature or solution viscosity, ${ }^{65}$ implying that it might be possible to modulate the extent of their mutual coupling. In addition, our hydrodynamic results supporting the influence of frictionmediated coupling in rotational deceleration suggest that, for intrinsically disordered regions connected to high-molecular weight proteins, the long-range motions are shifted strongly toward slower time scales, while the time scale of shorter range motions is probably less affected. This could potentially lead to a decrease in the extent of motional coupling and result in simplification of the dynamical analysis of intrinsically disordered regions of large protein systems.

\section{CONCLUSIONS}

In conclusion, the protein proton relaxation rate measurements reported here indicate that long-range correlated motions are an intrinsic property of disordered proteins. These motions are associated with residual order that is characterized by $S_{\mathrm{C}}{ }^{2}$ order parameters of about 0.1 and derive from nonperfect averaging of the dipolar interactions in the subnanosecond time scale. The slow reorientation of IDPs occurring beneath their largeamplitude fast internal motions is strongly influenced by hydrodynamic coupling among different protein segments and can be satisfactorily predicted on the basis of random coil conformer ensembles. The hydrodynamic coupling-mediated rotational deceleration offers a general physical picture for the presence of slow motions in IDPs irrespective of their local and long-range structural propensities. The rotational deceleration of protein segments in IDPs resembles the hydrodynamic coupling between interdomain motions and overall tumbling in flexible multidomain proteins ${ }^{39}$ and signifies the general importance of friction-mediated coupling in highly flexible macromolecular systems, for which temporal regulation of the activity of different modules is essential.

\section{ASSOCIATED CONTENT}

\section{S Supporting Information}

Supporting methods, table, and Figures $\mathrm{S} 1-\mathrm{S} 9$. This material is available free of charge via the Internet at http://pubs.acs.org.

\section{AUTHOR INFORMATION}

\section{Corresponding Authors}

luchinat@cerm.unifi.it

markus.zweckstetter@dzne.de

\section{Author Contributions}

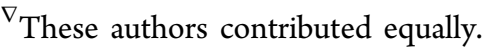

\section{Notes}

The authors declare no competing financial interest.

\section{ACKNOWLEDGMENTS}

This work was supported by Ente Cassa di Risparmio, MIURFIRB contracts RBLA032ZM7, RBRN07BMCT, by the German Science Foundation (ZW 71/7-1 to M.Z.) and by European Commission, contracts Bio-DNP 011721, WeNMR no. 261572, and Bio-NMR no. 261863. Eckhard Mandelkow and Jacek Biernat are acknowledged for providing samples of the Tau proteins and for discussions. Nikolai Skrynnikov is acknowledged for many extensive discussions. Marco Allegrozzi is acknowledged for providing the denatured lysozyme sample. We thank Dr. Eva-Maria Mandelkow and Dr. Francesca Munari for useful discussions, Frederik Klama for the Python script of HYCUD, Ilka Lindner for preparation of Tau samples, Karin Giller for preparation of $\alpha$-synuclein samples, and Tairan Yuwen for measuring ${ }^{15} \mathrm{~N}$ relaxation in $50 \% \mathrm{D}_{2} \mathrm{O}-50 \% \mathrm{H}_{2} \mathrm{O}$ sample of denatured ubiquitin.

\section{REFERENCES}

(1) Tompa, P. Trends Biochem. Sci. 2012, 37, 509.

(2) Metallo, S. J. Curr. Opin. Chem. Biol. 2010, 14, 481.

(3) Rezaei-Ghaleh, N.; Blackledge, M.; Zweckstetter, M. ChemBioChem 2012, 13, 930.

(4) Schneider, R.; Huang, J. R.; Yao, M.; Communie, G.; Ozenne, V.; Mollica, L.; Salmon, L.; Jensen, M. R.; Blackledge, M. Mol. Biosyst. 2012, 8, 58.

(5) Jensen, M. R.; Ruigrok, R. W.; Blackledge, M. Curr. Opin. Struct. Biol. 2013, 23, 426.

(6) Farrow, N. A.; Zhang, O.; Forman-Kay, J. D.; Kay, L. E. Biochemistry 1995, 34, 868.

(7) Farrow, N. A.; Zhang, O.; Forman-Kay, J. D.; Kay, L. E. Biochemistry 1997, 36, 2390.

(8) Farrow, N. A.; Zhang, O.; Szabo, A.; Torchia, D. A.; Kay, L. E. J. Biomol. NMR 1995, 6, 153.

(9) Rezaei-Ghaleh, N.; Giller, K.; Becker, S.; Zweckstetter, M. Biophys. J. 2011, 101, 1202.

(10) Shojania, S.; O’Neil, J. D. J. Biol. Chem. 2006, 281, 8347.

(11) Kaderavek, P.; Zapletal, V.; Rabatinova, A.; Krasny, L.; Sklenar, V.; Zidek, L. J. Biomol. NMR 2014, 58, 193.

(12) Buck, M.; Schwalbe, H.; Dobson, C. M. J. Mol. Biol. 1996, 257, 669.

(13) Buevich, A. V.; Shinde, U. P.; Inouye, M.; Baum, J. J. Biomol. NMR 2001, 20, 233. 
(14) Modig, K.; Poulsen, F. M. J. Biomol. NMR 2008, 42, 163.

(15) Prompers, J. J.; Bruschweiler, R. J. Am. Chem. Soc. 2002, 124, 4522

(16) Schwalbe, H.; Fiebig, K. M.; Buck, M.; Jones, J. A.; Grimshaw, S. B.; Spencer, A.; Glaser, S. J.; Smith, L. J.; Dobson, C. M. Biochemistry 1997, 36, 8977.

(17) Klein-Seetharaman, J.; Oikawa, M.; Grimshaw, S. B.; Wirmer, J.; Duchardt, E.; Ueda, T.; Imoto, T.; Smith, L. J.; Dobson, C. M.; Schwalbe, H. Science 2002, 295, 1719.

(18) Schwarzinger, S.; Wright, P. E.; Dyson, H. J. Biochemistry 2002, $41,12681$.

(19) Bertoncini, C. W.; Rasia, R. M.; Lamberto, G. R.; Binolfi, A.; Zweckstetter, M.; Griesinger, C.; Fernandez, C. O. J. Mol. Biol. 2007, 372, 708.

(20) Brutscher, B.; Bruschweiler, R.; Ernst, R. R. Biochemistry 1997, 36, 13043.

(21) Choy, W. Y.; Forman-Kay, J. D. J. Mol. Biol. 2001, 308, 1011.

(22) Choy, W. Y.; Kay, L. E. J. Am. Chem. Soc. 2003, 125, 11988.

(23) Dyson, H. J.; Wright, P. E. Nat. Rev. Mol. Cell Biol. 2005, 6, 197.

(24) Huang, J. R.; Grzesiek, S. J. Am. Chem. Soc. 2010, 132, 694.

(25) Plaxco, K. W.; Gross, M. Nat. Struct. Biol. 2001, 8, 659.

(26) Salmon, L.; Nodet, G.; Ozenne, V.; Yin, G.; Jensen, M. R.; Zweckstetter, M.; Blackledge, M. J. Am. Chem. Soc. 2010, 132, 8407.

(27) Shortle, D.; Ackerman, M. S. Science 2001, 293, 487.

(28) Wirmer, J.; Berk, H.; Ugolini, R.; Redfield, C.; Schwalbe, H. Protein Sci. 2006, 15, 1397.

(29) Bertini, I.; Gupta, Y. K.; Luchinat, C.; Parigi, G.; Schlorb, C.; Schwalbe, H. Angew. Chem., Int. Ed. Engl. 2005, 44, 2223.

(30) Ferrante, G.; Sykora, S. Adv. Inorg. Chem. 2005, 57, 405.

(31) Luchinat, C.; Parigi, G. J. Am. Chem. Soc. 2007, 129, 1055.

(32) Borsi, V.; Luchinat, C.; Parigi, G. Biophys. J. 2009, 97, 1765.

(33) Wirmer, J.; Schlorb, C.; Klein-Seetharaman, J.; Hirano, R.; Ueda, T.; Imoto, T.; Schwalbe, H. Angew. Chem., Int. Ed. Engl. 2004, 43, 5780.

(34) Lipari, G.; Szabo, A. J. Am. Chem. Soc. 1982, 104, 4546.

(35) Bertoncini, C. W.; Jung, Y. S.; Fernandez, C. O.; Hoyer, W.; Griesinger, C.; Jovin, T. M.; Zweckstetter, M. Proc. Natl. Acad. Sci. U.S.A. 2005, 102, 1430.

(36) Rasia, R. M.; Bertoncini, C. W.; Marsh, D.; Hoyer, W.; Cherny, D.; Zweckstetter, M.; Griesinger, C.; Jovin, T. M.; Fernandez, C. O. Proc. Natl. Acad. Sci. U.S.A. 2005, 102, 4294.

(37) Barghorn, S.; Davies, P.; Mandelkow, E. Biochemistry 2004, 43, 1694.

(38) Mukrasch, M. D.; Biernat, J.; von Bergen, M.; Griesinger, C.; Mandelkow, E.; Zweckstetter, M. J. Biol. Chem. 2005, 280, 24978.

(39) Rezaei-Ghaleh, N.; Klama, F.; Munari, F.; Zweckstetter, M. Angew. Chem., Int. Ed. Engl. 2013, 52, 11410.

(40) Bernado, P.; Bertoncini, C. W.; Griesinger, C.; Zweckstetter, M.; Blackledge, M. J. Am. Chem. Soc. 2005, 127, 17968.

(41) Receveur-Brechot, V.; Durand, D. Curr. Protein Pept. Sci. 2012, 13,55 .

(42) Ortega, A.; Amoros, D.; Garcia de la Torre, J. Biophys. J. 2011, 101, 892.

(43) Keepers, J. W.; James, T. L. J. Magn. Reson. 1984, 57, 404.

(44) Borgias, B. A.; Gochin, M.; Kerwood, D. J.; James, T. L. Prog. Nucl. Magn. Reson. Spectrosc. 1990, 22, 83.

(45) Bussell, R., Jr.; Eliezer, D. J. Biol. Chem. 2001, 276, 45996.

(46) Dedmon, M. M.; Lindorff-Larsen, K.; Christodoulou, J.; Vendruscolo, M.; Dobson, C. M. J. Am. Chem. Soc. 2005, 127, 476.

(47) Bertini, I.; Fragai, M.; Luchinat, C.; Parigi, G. Magn. Reson. Chem. 2000, 38, 543.

(48) Chen, K.; Tjandra, N. J. Am. Chem. Soc. 2008, 130, 12745.

(49) Munari, F.; Rezaei-Ghaleh, N.; Xiang, S.; Fischle, W.; Zweckstetter, M. PLoS One 2013, 8, e60887.

(50) Walsh, J. D.; Meier, K.; Ishima, R.; Gronenborn, A. M. Biophys. J. 2010, 99, 2636.

(51) Fatemi, N.; Korzhnev, D. M.; Velyvis, A.; Sarkar, B.; FormanKay, J. D. Biochemistry 2010, 49, 8468.
(52) Obolensky, O. I.; Schlepckow, K.; Schwalbe, H.; Solov'yov, A. V. J. Biomol. NMR 2007, 39, 1.

(53) Garcia De La Torre, J.; Huertas, M. L.; Carrasco, B. Biophys. J. 2000, 78, 719 .

(54) Bokor, M.; Csizmok, V.; Kovacs, D.; Banki, P.; Friedrich, P.; Tompa, P.; Tompa, K. Biophys. J. 2005, 88, 2030.

(55) Gallat, F. X.; Laganowsky, A.; Wood, K.; Gabel, F.; van Eijck, L.; Wuttke, J.; Moulin, M.; Hartlein, M.; Eisenberg, D.; Colletier, J. P.; Zaccai, G.; Weik, M. Biophys. J. 2012, 103, 129.

(56) Bernado, P.; Blanchard, L.; Timmins, P.; Marion, D.; Ruigrok, R. W.; Blackledge, M. Proc. Natl. Acad. Sci. U.S.A. 2005, 102, 17002.

(57) Mukrasch, M. D.; Bibow, S.; Korukottu, J.; Jeganathan, S.; Biernat, J.; Griesinger, C.; Mandelkow, E.; Zweckstetter, M. PLoS Biol. 2009, 7, e34.

(58) Jensen, M. R.; Markwick, P. R.; Meier, S.; Griesinger, C.; Zweckstetter, M.; Grzesiek, S.; Bernado, P.; Blackledge, M. Structure 2009, 17, 1169.

(59) Bertoncini, C. W.; Fernandez, C. O.; Griesinger, C.; Jovin, T. M.; Zweckstetter, M. J. Biol. Chem. 2005, 280, 30649.

(60) Sung, Y. H.; Eliezer, D. J. Mol. Biol. 2007, 372, 689.

(61) Mukrasch, M. D.; Markwick, P.; Biernat, J.; Bergen, M.; Bernado, P.; Griesinger, C.; Mandelkow, E.; Zweckstetter, M.; Blackledge, M. J. Am. Chem. Soc. 2007, 129, 5235.

(62) Eliezer, D.; Barre, P.; Kobaslija, M.; Chan, D.; Li, X.; Heend, L. Biochemistry 2005, 44, 1026.

(63) Xue, Y.; Skrynnikov, N. R. J. Am. Chem. Soc. 2011, 133, 14614.

(64) Halle, B. J. Chem. Phys. 2009, 131, 224507.

(65) Wu, K. P.; Baum, J. Biomol. NMR Assign. 2011, 5, 43. 